\title{
Combining Upper Limb Robotic Rehabilitation with Other Therapeutic Approaches after Stroke: Current Status, Rationale, and Challenges
}

\author{
Stefano Mazzoleni, ${ }^{1,2}$ Christophe Duret, ${ }^{3,4}$ Anne Gaëlle Grosmaire, ${ }^{3}$ and Elena Battini ${ }^{1,2}$ \\ ${ }^{1}$ The BioRobotics Institute, Scuola Superiore Sant'Anna, Pisa, Italy \\ ${ }^{2}$ Rehabilitation Bioengineering Laboratory, Volterra, Italy \\ ${ }^{3}$ Centre de Rééducation Fonctionnelle Les Trois Soleils, Médecine Physique et de Réadaptation, Unité de Neurorééducation, \\ Boissise-Le-Roi, France \\ ${ }^{4}$ Centre Hospitalier Sud Francilien, Neurologie, Corbeil-Essonnes, France
}

Correspondence should be addressed to Stefano Mazzoleni; stefano.mazzoleni@santannapisa.it

Received 3 January 2017; Revised 12 July 2017; Accepted 10 August 2017; Published 13 September 2017

Academic Editor: Erwin van Wegen

Copyright (c) 2017 Stefano Mazzoleni et al. This is an open access article distributed under the Creative Commons Attribution License, which permits unrestricted use, distribution, and reproduction in any medium, provided the original work is properly cited.

\begin{abstract}
A better understanding of the neural substrates that underlie motor recovery after stroke has led to the development of innovative rehabilitation strategies and tools that incorporate key elements of motor skill relearning, that is, intensive motor training involving goal-oriented repeated movements. Robotic devices for the upper limb are increasingly used in rehabilitation. Studies have demonstrated the effectiveness of these devices in reducing motor impairments, but less so for the improvement of upper limb function. Other studies have begun to investigate the benefits of combined approaches that target muscle function (functional electrical stimulation and botulinum toxin injections), modulate neural activity (noninvasive brain stimulation), and enhance motivation (virtual reality) in an attempt to potentialize the benefits of robot-mediated training. The aim of this paper is to overview the current status of such combined treatments and to analyze the rationale behind them.
\end{abstract}

\section{Introduction}

Significant advances have been made in the management of stroke (including prevention, acute management, and rehabilitation); however cerebrovascular diseases remain the third most common cause of death and the first cause of disability worldwide [1-6]. Stroke causes brain damage, leading to loss of motor function. Upper limb (UL) function is particularly reduced, resulting in disability. Many rehabilitation techniques have been developed over the last decades to facilitate motor recovery of the UL in order to improve functional ability and quality of life [7-10]. They are commonly based on principles of motor skill learning to promote plasticity of motor neural networks. These principles include intensive, repetitive, task-oriented movement-based training [11-19]. A better understanding of the neural substrates of motor relearning has led to the development of innovative strategies and tools to deliver exercise that meets these requirements. Treatments mostly target the neurological impairment (paresis, spasticity, etc.) through the activation of neural circuits or by acting on peripheral effectors. Robotic devices provide exercises that incorporate key elements of motor learning. Advanced robotic systems can offer highly repetitive, reproducible, interactive forms of training for the paretic limb, which are quantifiable. Robotic devices also enable easy and objective assessment of motor performance in standardized conditions by the recording of biomechanical data (i.e., speed, forces) [20-22]. This data can be used to analyze and assess motor recovery in stroke patients [23-26]. Since the 1990s, many other technology-based approaches and innovative pharmaceutical treatments have also been developed for rehabilitation, including virtual reality- (VR-) based systems, botulinum neurotoxin (BoNT) injections, and noninvasive brain stimulation (NIBS) (Direct Current Stimulation (tDCS) 
and repetitive transcranial magnetic stimulation (rTMS)). There is currently no high-quality evidence to support any of these innovative interventions, despite the fact that some are used in routine practice [27]. By their respective mechanisms of action, each of these treatments could potentiate the effects of robotic therapy, leading to greater improvements in motor capacity. The aim of this paper is to review studies of combined treatments based on robotic rehabilitation and to analyze the rationale behind such approaches.

\section{Robot-Assisted Upper Limb Rehabilitation after Stroke: Two Decades of Evidence and Misunderstanding}

Robotic systems for upper limb rehabilitation have two main designs: (i) exoskeletons, generally based on torque actuators that control each joint of the affected limb to be treated and (ii) end-effectors systems that guide only the most distal part of the affected limb [28-30].

During the last 2 decades, a growing number of robotic devices have been developed (e.g., MIME, ARMin, MITMANUS, and NeReBot) to offer intensive training based on repeated movements and challenging task-specific exercises [31]. These devices also provide different forms of sensorimotor feedback to patients, which can positively influence the training outcome [32].

Several systematic reviews and meta-analyses have been carried out on the numerous studies of robotic rehabilitation to assess the effects in patients with stroke with a growing interest in the latest years [32-37].

Despite significant heterogeneity in the types of system evaluated (e.g., distal or proximal UL rehabilitation) as well as the clinical research paradigms used [33], there is a general consensus that robot-assisted upper limb therapy is safe and significantly reduces motor impairment of the limb segments targeted by the robotic device (mainly the shoulder and elbow). However, improvements in motor function, although significant, tend to be small [36]. Moreover, some results suggest that it is the addition of robotic therapy to conventional treatment (CT) that is particularly effective $[32,34]$ although the effects also depend on the stage at which the therapy is carried out; Bertani et al. [37] and Zhang et al. [32] found that, in patients with chronic stroke, robotic training was more effective in reducing motor impairment than conventional therapy, but not in patients with acute stroke.

Most reviews concluded that robotic therapy does not provide any functional benefit and so does not improve activities of daily living due to the lack of generalization of improvement to untreated joints (especially the wrist and hand). However the latest update of the review conducted by Mehrholz et al. [35] found that, compared to other interventions, robot-assisted arm training may improve activities of daily living in the acute phase after stroke but not in the chronic phase [35]; however they concluded that the quality of evidence was low to very low.

The effect of robotic therapy on muscle tone remains uncertain as only two reviews included this outcome; Bertani et al. [37] found no change while Veerbeek et al. [36] showed a negative effect of robotic therapy on muscle tone.

One raised question is that of the effect of the robotic system itself on improvements in motor outcomes versus simply the provision of highly repetitive treatment. Current evidence suggests it is the large number of repetitions that is effective since there are no differences in outcome between robot-assisted therapy and dose-matched conventional therapy [34]. In the future, robotic systems may become more effective by the use of specific robotic paradigms such as perturbing forces that enhance movement errors. Preliminary studies suggest that such paradigms appear more effective than assistive and simply repetitive practice $[38,39]$.

Thus two decades after the pioneering study by Aisen et al. [40], a sufficient body of evidence suggests that robot-assisted upper limb rehabilitation improves motor impairment. The effect on muscle overactivity requires further study. It has yet to be established if improvements in function result from generalization of improvements to untrained limb segments. Some reviews suggest that greater functional improvements occur when robotic rehabilitation is carried out in the subacute phase of stroke.

Robotic systems should therefore be considered as vehicles that enable delivery of evidence-based, impairmentoriented treatment, providing highly repetitive, intensive, and interactive treatment that is not possible in usual care. Further improvements in function might come from combining treatments that target different impairments (e.g., weakness and spasticity) and other components of the neural disorder (e.g., interhemispheric imbalance) or are based on a more functional approach (virtual reality, FES).

\section{Robotic Systems Combined with Other Therapeutic Innovations after Stroke: A General Overview}

3.1. Functional Electrical Stimulation. The effects of functional electrical stimulation (FES) on upper limb recovery in individuals with stroke have been extensively investigated during the last 30 years. FES activates muscles in a pattern that produces a functionally useful movement [41]. Most FES systems can stimulate up to three specific muscle groups in the upper limb, and studies have shown that this can facilitate recovery of functional reach and grasp movements [42].

The effects of FES on motor function are mixed in the literature. A meta-analysis of 10 randomized controlled trials (RCT) concluded that the addition of FES to conventional therapy did not further improve motor function [43]. In contrast, two systematic reviews found that the addition of FES to a motor training program has a greater effect on improving upper limb functional abilities than training alone or no rehabilitation [44], especially when applied in the subacute phase [45]. The effects may depend on the severity of the impairment. Studies have shown that the addition of FES to usual rehabilitation improves motor function in patients with mild/moderate UL paresis [46] but not severe impairment 
[47]. Moreover, some studies have suggested that FES is more effective if it is triggered by voluntary muscle contractions (EMG-initiated FES) [48].

These results stimulated the development of hybrid robotic systems combining FES with robotic systems [49]. This is particularly logical since most UL robotic rehabilitation devices only train movements of the shoulder and elbow; thus the association with FES of the hand muscles provides simultaneous, functional training to the whole upper limb. A proof of concept study [50] showed functional improvements on the ARAT scale [51] and the ABILHAND questionnaire [52] following training with a robotic device combined with FES. Another study used FES to facilitate active participation in a virtual reality tracking task by stimulating the deltoid and triceps muscles while the arm was supported by a robot. Although the study was carried out in only 5 patients and was not controlled, the results are encouraging, showing a reduction of motor impairment (Fugl-Meyer score, FM score) [53], improvement in tracking capacity, and a reduction in the need for FES over the course of treatment [54]. A randomized controlled trial in patients with chronic stroke [55] found that a robotic device driven by EMG that provided FES to the wrist was more effective in improving FM scores and manual abilities [51] than robotic therapy alone. More recently, Miyasaka et al. [56] showed that FES of the anterior deltoid and triceps brachii associated with shoulder/elbow robotic training increased range of motion and potentially improved the effectiveness of the robot-assisted rehabilitation. Resquín et al. [57] published a comprehensive description of current hybrid approaches and clinical assessments and concluded that results were promising when the assistance is provided to the distal segments (Wrist/hand) together with the proximal joints.

\subsection{Repetitive Transcranial Magnetic Stimulation (rTMS).}

Transcranial magnetic stimulation (TMS) is a 25 -year-old noninvasive technique used to characterize the physiological processes involved in functional consequences of stroke [58]. The use of repetitive transcranial magnetic stimulation (rTMS) for therapeutic purposes is a relatively recent approach. rTMS involves indirect activation of corticospinal cells via a coil placed over the motor cortex, through which a brief high current is passed [59]. It is now well established that low frequency $(1 \mathrm{~Hz})$ rTMS has inhibitory effects on the motor cortex and high frequency rTMS has an excitatory effect $(5 \mathrm{~Hz}$ or more) [60]. Thus, rTMS can be used to treat interhemispheric imbalance [60]. Several studies have suggested that the beneficial effect of rTMS is more marked in subcortical rather than cortical stroke [61, 62].

Along with clinical evaluation, functional magnetic resonance imaging (fMRI), and diffusion tensor imaging, rTMS can be used as a predictor of upper limb motor recovery after stroke [63]. Moreover, it has been shown to predict individuals who are more likely to benefit from robot-based therapy [64] since increases in Box and Block Test (BBT) scores (functional outcome measure) after robotic training have been shown to be correlated with a lower baseline motor evoked potential (MEP) amplitude on rTMS.
A study of six patients with chronic stroke highlighted the need to adapt rTMS to the patient's lesions since rTMS may have different effects on motor reorganization depending on the location of the lesions [65]. A group of European experts found a sufficient body of evidence (level B) to suggest low frequency rTMS of contralesional M1 is effective in improving motor outcomes in patients with chronic stroke [66].

A growing number of studies have investigated the effects of combining various upper limb rehabilitation techniques with rTMS [67-71], with inconclusive results. A recent systematic review with meta-analysis [72] showed that rTMS combined with upper limb training has no additional effect on motor function when compared to upper limb training alone. Hosomi et al. [73] found a modest improvement in patients with subacute stroke following rTMS associated with conventional rehabilitation.

However, despite these mixed results, it seems likely that the association of the relative normalization of cortical excitability by rTMS, with repetitive active robotic training, would potentiate the effects of each treatment, resulting in greater motor recovery [74]. Currently, only a few studies [64] have evaluated the combined effect of rTMS and robotic rehabilitation for the upper limb. However the results are too preliminary to conclude.

3.3. Transcranial Direct Current Stimulation (tDCS). Transcranial Direct Current Stimulation (tDCS) is a noninvasive, painless neural modulation technique. It involves cortical stimulation by a constant and low intensity current delivered via two electrodes placed over the head. Anodal and cathodal tDCS have different effects on the motor cortex. The former has an excitatory effect while the latter inhibits or reduces neuronal activity. Knowledge of these effects is useful as general rule; however numerous other factors also affect inhibition and excitation [75], such as axonal orientation [76]. Several studies have demonstrated that anodal tDCS effectively increases activation of the primary motor cortex [77, 78].

The application of cathodal tDCS over the unaffected motor cortex has been shown to improve the motor control of the proximal upper limb in the case of mild impairment and to worsen control in the case of moderate to severe impairment. It is likely that difference in effect is related to the level of spasticity [79]. Moreover, when used as an adjunct to physical therapy, cathodal tDCS significantly reduces muscle tone and improves activities of daily living [80]. Several studies based on a single-session of cathodal tDCS in patients with chronic stroke showed improved motor performance of the paretic hand [81] and finger movement tasks [82]. However, no evidence was found to support either cathodal tDCS over the contralesional motor cortex or anodal tDCS over the ipsilesional motor cortex [75].

Several studies have investigated the effects of combining tDCS with robot therapy. Edwards et al. [83] showed that raised corticospinal excitability accompanied by reduced cortical inhibition following anodal tDCS persisted during task-specific robotic wrist training, confirming the rational for combining the treatments. However, a randomized trial 
found no additional effect of tDCS combined with bilateral robotic training in subacute stroke, either with anodal or cathodal tDCS [84]. Equally, Triccas et al. [85] found no further effect of robotic training on upper limb motor function with the association of tDCS. A recent literature analysis found that the addition of tDCS (unilateral anodal or cathodal or bilateral) to robotic training (unilateral or bilateral, distal or proximal) did not result in greater improvements than robotic therapy alone in either the subacute or chronic phase [86].

The timing of the application of tDCS may be important. One study showed that movement smoothness improved only when anodal tDCS was delivered prior to robotic training, rather than during or after robotic training [87]. The phase of stroke may also be important. A recent study demonstrated that bilateral tDCS combined with upper extremity robot-assisted therapy resulted in greater improvements in patients with chronic and subcortical stroke than patients with acute and cortical stroke [88].

It appears that the effects of anodal tDCS (on the affected hemisphere) and cathodal tDCS (on the unaffected hemisphere) combined with robot-assisted arm training are similar. A comparative study in patients with severe impairment found that spasticity and motor function (upper limb section of the Fugl-Meyer Assessment (FMA) scale) improved to a similar extent with both modalities [89].

3.4. Virtual Reality. Virtual reality (VR) is an innovative, interactive, and adaptive treatment modality. It can be used to provide sensorimotor training in complex, enriched environments, which would be impossible to design in the real world. VR optimizes patient engagement and can provide mass practice tailored to the individual [90]. VR is considered an important patient-centered tool for stroke rehabilitation because of its characteristics, such as contextualized environments and task-oriented training [91, 92]. Moreover, patients with stroke use similar movement strategies in virtual reality environments as in the natural world $[93,94]$. VR can also be used as a research tool to investigate how patients interact with different environments in realistic conditions, allowing a precise evaluation with varying stimuli.

The results of several meta-analyses and systematic reviews suggest that the use of virtual reality as an adjunct to usual care can lead to substantial improvements in upper limb motor function and in activities of daily living when compared with the same dose of conventional care in patients with stroke $[95,96]$. A recent review found that VR has positive effects in terms of body function and body structure and that the effects are mainly related to the upper limb. However, the effects on activity and participation are small [97].

Several groups have developed systems that combine VR with robotic devices $[98,99]$. The robots used are mostly exoskeletons with passive gravity compensation [100] or assist-as-needed algorithms [101]. The common goal is to optimize engagement in assistive therapy, providing patients with continuous visual feedback on movement quality through kinematic modeling [100]. This technology is novel; therefore no randomized, controlled trials have been carried out; however preliminary studies have shown improvements in motor performance of reaching tasks to visual targets [102] and in manipulation tasks involving a hand-wrist assistive device [103]. Moreover, the latter study also demonstrated increased activation of the sensorimotor cortex during performance of grasp tasks, using functional MRI. Preliminary studies of novel applications combining VR-robots and instrumented gloves [104] suggest that specially designed virtual environments might activate the neural circuits involved in motor skill learning processes by the provision of modified visual feedback. Training finger individuation using a mechatronic-virtual reality system has been demonstrated to be more effective than dose-matched occupational therapy in chronic stroke patients [105] and adaptive robot-mediated training combined with a virtual learning environment has been shown to improve coordination in patients with chronic stroke [106].

3.5. Botulinum Toxin. Botulinum neurotoxin (BoNT) is a microbial protein that blocks acetylcholine release at the neuromuscular junction. It is used to reduce dystonia, spasticity, and related disorders. Over the last 2 decades, two BoNT serotypes (A and B) have become widely used in neurorehabilitation. BoNT has been demonstrated to be safe and effective for the treatment of spasticity in adults and children, including upper limb spastic paresis due to stroke and traumatic brain injury (TBI) [107-110].

BoNT injected in upper limb muscles reduces muscle tone, with perceived functional benefits [109]. Some studies have also shown that BoNT treatment is effective in decreasing cocontraction of antagonist muscles, facilitating agonist recruitment and increasing active range of motion [111]. However, improvements in active upper limb function remain to be demonstrated [110]. No improvements have been found on the Barthel Index [112], the Functional Independence Measure (FIM) [113], or measures of quality of life such as the SF-36 [114], particularly in early stages after stroke [115]. This lack of effect of the reduction of spasticity on active function suggests that BoNT injections should be combined with exercise therapy [115].

BoNT and robotic rehabilitation appear to be a natural, synergistic combination since several studies have demonstrated that repetitive movement-based robot therapy can reduce muscle tone as well as motor impairment [116-120]. This combination has been evaluated in a small number of studies in children with cerebral palsy (CP) [121, 122] and in patients with stroke [123-125]. A case study in children with cerebral palsy showed improvements in upper limb coordination and quality of motor performance [121]. Another study compared the effects of upper limb robotic therapy with a combined schedule (same robot-assisted training following BoNT injections) and showed a greater decrease in spasticity in the combined group [124]. Moreover, robotic devices are useful for the evaluation of the effects of BoNT. Kinematic assessment of six children with hemiplegia after BoNT administration using a robotic device showed significant improvements in accuracy and smoothness, which was correlated with the results of clinical scales [122]. 


\section{Discussion}

Over the last two decades, neurorehabilitation has evolved from being empirically based towards a more evidence-based form of practice, transferring the scientific concepts of neuroplasticity and motor relearning as well as advanced knowledge from clinical studies, into clinical practice. A growing body of evidence has demonstrated that stroke inducedchanges in cortical neuronal activity and neural circuits can be influenced by physical interventions that target functional deficits and/or impairments such as weakness and spasticity. A growing number of treatment methods and devices are becoming available to facilitate plastic-reorganization of the central nervous system. However, the optimization of treatment effectiveness might require combined approaches with complementary actions. The following section discusses the rationale for combining robotic therapy with other innovative approaches.

4.1. Priming Motor Learning-Based Processes. Motor recovery and/or improvements in motor performance after stroke are driven by motor skill leaning that can be mediated by movement-based rehabilitation paradigms. Robotic rehabilitation systems fully meet the requirements for motor learning, since they facilitate practice-dependent improvements in motor performance that persist over time. Although robust evidence for neurorehabilitation-induced plasticity is lacking, it has been suggested that robot-mediated treatment using adaptive algorithms (assistance or perturbation) might have the potential to enhance neuroplasticity [126].

Noninvasive brain stimulation (NIBS) techniques can transiently modulate brain excitability. An obvious application is to prime motor networks [127], increasing responsiveness to motor learning therapies, particularly by delivering them prior to movement-based training. The association of NIBS and robotic systems has been evaluated in preliminary studies with encouraging results $[88,128]$. However, many questions still remain with regard to the optimal use of NIBS alone (e.g., delivery of stimulation to the affected or unaffected hemisphere). These questions should be answered before investigating combined approaches.

One of the aims of combining virtual reality environments with robotic rehabilitation is to enhance patient motivation and engagement in movement-based practice. The association of VR with robot therapy adds a "recreational" component to rehabilitation that distracts patients from fatigue and pain and motivates them to improve their performance [129]. As such, many robots are equipped with user-friendly graphical interfaces to provide games-based exercises. However, to date, engineers have primarily focused on robot control strategies and mechanical issues rather than on interfaces and program content. Thus little attention has been paid to the contribution of the "VR" component of robotic therapy to improvements in performance. The virtual reality interface can provide immediate nonspecific visual and auditory feedback that has been shown to enhance motor performance when added to robotic training [130, 131]. Another interesting potential for VR-robot therapy is the provision of feedback on limb position and on potential movement errors. Grimm and Gharabaghi [100] found that rehabilitation with an exoskeleton combined with a virtual reality-based interface that displays a representation of the user arm can enhance performance on clinical tests as well as improving movement kinematics. This is supported by the fact that skill reacquisition is facilitated by feedback regarding knowledge of performance (attention directed to movement kinematics) more than knowledge of results, in which the goal is to achieve the movement, regardless of the strategy [132]. However, much work remains to be done in this field as evidence for the clinical effectiveness of VRrobotic rehabilitation is lacking.

4.2. Facilitating Agonist Muscle Recruitment. Although BoNT injections have been shown to reduce muscle spasticity, studies have failed to find a concomitant improvement in motor function and disability. It is likely that patients "learn" new patterns of movement in order to function as effectively as possible in the presence of spasticity. Once these new patterns are established, they may not change, even if spasticity is reduced. Repetitive, goal-directed movements using robotic devices may change these patterns, facilitating more normal movement and improving functional capacity, particularly since robot-mediated training has been shown to change muscle synergies [133]. However, this remains speculative as evidence is lacking.

FES has obvious applications to facilitate appropriate muscle recruitment, and its use with assist-as needed robotic rehabilitation appears to be an interesting combination. Although studies suggest that robot-mediated therapy should focus more on movement coordination than on muscle activation to be effective [134], EMG driven FES may be appropriate to potentiating active movement, especially in patients with severe impairment. Stimulation of both peripheral effectors and centrally controlled motor planning networks might facilitate motor recovery; however, again, this hypothesis needs to be investigated.

4.3. Combining Both Proximal and Distal Training. Currently available robotic devices are mostly single or bijoint-based modules that carry out training of only proximal or distal segments, with a single goal to improve either reaching or grasping ability. Hybrid systems combining FES with robot training can train both proximal and distal segments simultaneously, combining impairment-based therapy with functional-oriented tasks (reach-to-grasp). Although some studies have suggested there are no additional advantages when shoulder/elbow robot-assisted training is combined with functional tasks [135], this approach might need further development from both a technological and a clinical point of view. It is well established that voluntary movements are internally represented as goal-oriented motor actions, rather than movements of segments. It is therefore pertinent to rehabilitate arm and hand function together in a coordinated manner [136].

4.4. Optimizing Challenge and Motor Behavior. Engagement in rehabilitation is essential to promote learning [137]. VR and robotic systems require interaction by the patient, enhancing 
engagement as well as providing a focus on motor tasks. One of the underlying mechanisms is likely the rewards associated with actions; evidence indicates that reward is an effective feedback signal for the regulation of behavior and motor learning $[138,139]$ and has been shown to enhance motor control after robotic training [140]. VR provides an excellent framework for reward presentation [126]. While robotic control systems mostly use assistive algorithms, the association with a VR games-based program may optimize motor behavior, encouraging the patient to improve his/her own motor performance. However, the specific effects of VR on upper limb robotic training have not been studied.

\subsection{Robotic Devices for Evaluation: A Complement to Clinical} Scales. One advantage of robotic devices is their ability to provide a simple and affordable measurement of motion kinematics in standardized conditions, complementing clinical scales of motor function, with a high resolution and specific metrics [20-22]. Advanced systems can measure and record the kinematics of upper limb trajectories in order to determine indicators of movement performance. Kinematic variables provide a valuable measure of motor performance and movement quality. The utility of robotic devices for evaluation purposes has been demonstrated for the assessment of the effects of BoNT in both children with spastic hemiplegia $[122,141,142]$ and patients with stroke $[143,144]$. Moreover, kinematic parameters better characterize changes in active motor function after BoNT treatment than clinical scales (e.g., movement velocity and smoothness). However, more work is needed to optimize the use of robot-based measurements in clinical practice, including their use in decision making, and for treatment planning and progression.

\section{Conclusion}

There is currently some evidence to suggest that combination-therapies may be more effective than individual treatment techniques.

This paper highlights the potential benefits of combining upper limb movement-based robotic therapy with other approaches. Much work is, however, required to evaluate and optimize such combination-approaches as current studies have many limitations (small sample sizes, heterogeneity of technological devices and methodological paradigms used).

\section{Conflicts of Interest}

The authors have no conflicts of interest to declare.

\section{References}

[1] P. L. Kolominsky-Rabas, M. Weber, O. Gefeller, B. Neundoerfer, and P. U. Heuschmann, "Epidemiology of ischemic stroke subtypes according to TOAST criteria: incidence, recurrence, and long-term survival in ischemic stroke subtypes: a populationbased study," Stroke, vol. 32, no. 12, pp. 2735-2740, 2001.

[2] G. Kwakkel, B. J. Kollen, and R. C. Wagenaar, "Long term effects of intensity of upper and lower limb training after stroke: a randomised trial," Journal of Neurology Neurosurgery and Psychiatry, vol. 72, no. 4, pp. 473-479, 2002.

[3] P. Langhorne, J. Bernhardt, and G. Kwakkel, "Stroke rehabilitation," The Lancet, vol. 377, no. 9778, pp. 1693-1702, 2011.

[4] J. S. Balami and A. M. Buchan, "Complications of intracerebral haemorrhage," The Lancet Neurology, vol. 11, no. 1, pp. 101-118, 2012.

[5] F. Chollet and J.-F. Albucher, "Strategies to augment recovery after stroke," Current Treatment Options in Neurology, vol. 14, no. 6, pp. 531-540, 2012.

[6] M. A. Taqi, N. Vora, R. C. Callison, R. Lin, and T. J. Wolfe, "Past, present, and future of Endovascular stroke therapies," Neurology, vol. 79, no. 13, pp. S213-S220, 2012.

[7] P. Langhorne, F. Coupar, and A. Pollock, "Motor recovery after stroke: a systematic review," The Lancet Neurology, vol. 8, no. 8, pp. 741-754, 2009.

[8] B. B. Johansson, "Current trends in stroke rehabilitation. A review with focus on brain plasticity," Acta Neurologica Scandinavica, vol. 123, no. 3, pp. 147-159, 2011.

[9] K. N. Arya, S. Pandian, R. Verma, and R. K. Garg, "Movement therapy induced neural reorganization and motor recovery in stroke: a review," Journal of Bodywork and Movement Therapies, vol. 15, no. 4, pp. 528-537, 2011.

[10] L. Brewer, F. Horgan, A. Hickey, and D. Williams, "Stroke rehabilitation: recent advances and future therapies," QJM: An International Journal of Medicine, vol. 106, no. 1, pp. 11-25, 2013.

[11] F. Buma, G. Kwakkel, and N. Ramsey, "Understanding upper limb recovery after stroke," Restorative Neurology and Neuroscience, vol. 31, no. 6, pp. 707-722, 2013.

[12] C. Bütefisch, H. Hummelsheim, P. Denzler, and K.-H. Mauritz, "Repetitive training of isolated movements improves the outcome of motor rehabilitation of the centrally paretic hand," Journal of the Neurological Sciences, vol. 130, no. 1, pp. 59-68, 1995.

[13] E. V. Cooke, K. Mares, A. Clark, R. C. Tallis, and V. M. Pomeroy, "The effects of increased dose of exercise-based therapies to enhance motor recovery after stroke: a systematic review and meta-analysis," BMC Medicine, vol. 8, article 60, 2010.

[14] H. Feys, W. de Weerdt, and G. Verbeke, "Early and repetitive stimulation of the arm can substantially improve the long-term outcome after stroke: a 5-year follow-up study of a randomized trial," Stroke, vol. 35, no. 4, pp. 924-929, 2004.

[15] G. Kwakkel, R. C. Wagenaar, J. W. R. Twisk, G. J. Lankhorst, and J. C. Koetsier, "Intensity of leg and arm training after primary middle-cerebral-artery stroke: a randomised trial," The Lancet, vol. 354, no. 9174, pp. 191-196, 1999.

[16] G. Kwakkel, B. Kollen, and E. Lindeman, "Understanding the pattern of functional recovery after stroke: facts and theories," Restorative Neurology and Neuroscience, vol. 22, no. 3-4, pp. 281299, 2004.

[17] N. B. Lincoln, R. H. Parry, and C. D. Vass, "Randomized, controlled trial to evaluate increased intensity of physiotherapy treatment of arm function after stroke," Stroke, vol. 30, no. 3, pp. 573-579, 1999.

[18] G. Kwakkel, R. van Peppen, R. C. Wagenaar et al., "Effects of augmented exercise therapy time after stroke: a meta-analysis," Stroke, vol. 35, no. 11, pp. 2529-2536, 2004.

[19] J. M. Veerbeek, E. van Wegen, R. van Peppen et al., "What is the evidence for physical therapy poststroke? a systematic review and meta-analysis," PLoS ONE, vol. 9, no. 2, Article ID e87987, 2014. 
[20] H. I. Krebs, M. L. Aisen, B. T. Volpe, and N. Hogan, "Quantization of continuous arm movements in humans with brain injury," Proceedings of the National Academy of Sciences of the United States of America, vol. 96, no. 8, pp. 4645-4649, 1999.

[21] L. Zollo, L. Rossini, M. Bravi, G. Magrone, S. Sterzi, and E. Guglielmelli, "Quantitative evaluation of upper-limb motor control in robot-aided rehabilitation," Medical and Biological Engineering and Computing, vol. 49, no. 10, pp. 1131-1144, 2011.

[22] M. Gilliaux, T. M. Lejeune, C. Detrembleur et al., "Using the robotic device REAplan as a valid, reliable, and sensitive too 1 to quantify upper limb impairments in stroke patients," Journal of Rehabilitation Medicine, vol. 46, no. 2, pp. 117-125, 2014.

[23] S. Mazzoleni, P. Sale, M. Tiboni, M. Franceschini, M. C. Carrozza, and F. Posteraro, "Upper limb robot-assisted therapy in chronic and subacute stroke patients: a kinematic analysis," American Journal of Physical Medicine and Rehabilitation, vol. 92, Supplement 1, no. 10, pp. e26-e37, 2013.

[24] H. I. Krebs, M. Krams, D. K. Agrafiotis et al., "Robotic measurement of arm movements after stroke establishes biomarkers of motor recovery," Stroke, vol. 45, no. 1, pp. 200-204, 2014.

[25] C. Shirota, J. Jansa, J. Diaz et al., "On the assessment of coordination between upper extremities: towards a common language between rehabilitation engineers, clinicians and neuroscientists," Journal of NeuroEngineering and Rehabilitation, vol. 13, no. 1, article 80, 2016.

[26] C. Duret, O. Courtial, and A. G. Grosmaire, "Kinematic measures for upper limb motor assessment during robot-mediated training in patients with severe sub-acute stroke," Restorative Neurology and Neuroscience, vol. 34, no. 2, pp. 237-245, 2016.

[27] A. Pollock, S. E. Farmer, M. C. Brady et al., "Interventions for improving upper limb function after stroke," The Cochrane Database of Systematic Reviews, no. 11, p. CD010820, 2014.

[28] R. Riener, T. Nef, and G. Colombo, "Robot-aided neurorehabilitation of the upper extremities," Medical and Biological Engineering and Computing, vol. 43, no. 1, pp. 2-10, 2005.

[29] R. C. V. Loureiro, W. S. Harwin, K. Nagai, and M. Johnson, "Advances in upper limb stroke rehabilitation: a technology push," Medical and Biological Engineering and Computing, vol. 49, no. 10, pp. 1103-1118, 2011.

[30] W. H. Chang and Y.-H. Kim, "Robot-assisted therapy in stroke rehabilitation," Journal of Stroke, vol. 15, no. 3, pp. 174-181, 2013.

[31] F. Yakub, A. Z. Md. Khudzari, and Y. Mori, "Recent trends for practical rehabilitation robotics, current challenges and the future," International Journal of Rehabilitation Research, vol. 37, no. 1, pp. 9-21, 2014.

[32] C. Zhang, C. W. Li-Tsang, and R. K. Au, "Robotic approaches for the rehabilitation of upper limb recovery after stroke: a systematic review and meta-analysis," International Journal of Rehabilitation Research, vol. 40, no. 1, pp. 19-28, 2017.

[33] G. Kwakkel, B. J. Kollen, and H. I. Krebs, "Effects of robotassisted therapy on upper limb recovery after stroke: a systematic review," Neurorehabilitation and Neural Repair, vol. 22, no. 2, pp. 111-121, 2008.

[34] N. Norouzi-Gheidari, P. S. Archambault, and J. Fung, "Effects of robot-assisted therapy on stroke rehabilitation in upper limbs: systematic review and meta-analysis of the literature," Journal of Rehabilitation Research and Development, vol. 49, no. 4, pp. 479-496, 2012.

[35] J. Mehrholz, M. Pohl, T. Platz, J. Kugler, and B. Elsner, "Electromechanical and robot-assisted arm training for improving activities of daily living, arm function, and arm muscle strength after stroke," Cochrane Database of Systematic Reviews, no. 11, p. CD006876, 2015.

[36] J. M. Veerbeek, A. C. Langbroek-Amersfoort, E. E. H. Van Wegen, C. G. M. Meskers, and G. Kwakkel, "Effects of robotassisted therapy for the upper limb after stroke," Neurorehabilitation and Neural Repair, vol. 31, no. 2, pp. 107-121, 2017.

[37] R. Bertani, C. Melegari, M. C. De Cola, A. Bramanti, P. Bramanti, and R. S. Calabrò, "Effects of robot-assisted upper limb rehabilitation in stroke patients: a systematic review with metaanalysis," Neurological Sciences, 2017.

[38] J. L. Patton, M. E. Stoykov, M. Kovic, and F. A. Mussa-Ivaldi, "Evaluation of robotic training forces that either enhance or reduce error in chronic hemiparetic stroke survivors," Experimental Brain Research, vol. 168, no. 3, pp. 368-383, 2006.

[39] F. Abdollahi, E. D. Case Lazarro, and M. Listenberger, "Error augmentation enhancing arm recovery in individuals with chronic stroke: a randomized crossover design," Neurorehabilitation and Neural Repair, vol. 28, pp. 120-128, 2014.

[40] M. L. Aisen, H. I. Krebs, N. Hogan, F. McDowell, and B. T. Volpe, "The effect of robot-assisted therapy and rehabilitative training on motor recovery following stroke," Archives of Neurology, vol. 54, no. 4, pp. 443-446, 1997.

[41] O. Schuhfried, R. Crevenna, V. Fialka-Moser, and T. Paternostro-Sluga, "Non-invasive neuromuscular electrical stimulation in patients with central nervous system lesions: an educational review," Journal of Rehabilitation Medicine, vol. 44, no. 2, pp. 99 $105,2012$.

[42] K. Meadmore, T. Exell, C. Freeman et al., "Electrical stimulation and iterative learning control for functional recovery in the upper limb post-stroke," in Proceedings of the 2013 IEEE 13th International Conference on Rehabilitation Robotics, ICORR '13, vol. 2013, June 2013.

[43] A. K. Vafadar, J. N. Côté, and P. S. Archambault, "Effectiveness of functional electrical stimulation in improving clinical outcomes in the upper arm following stroke: a systematic review and meta-analysis," BioMed Research International, vol. 2015, Article ID 729768, 14 pages, 2015.

[44] O. A. Howlett, N. A. Lannin, L. Ada, and C. Mckinstry, "Functional electrical stimulation improves activity after stroke: a systematic review with meta-analysis," Archives of Physical Medicine and Rehabilitation, vol. 96, no. 5, Article ID 56090, pp. 934-943, 2015.

[45] J. Eraifej, W. Clark, B. France, S. Desando, and D. Moore, "Effectiveness of upper limb functional electrical stimulation after stroke for the improvement of activities of daily living and motor function: a systematic review and meta-analysis," Systematic Reviews, vol. 6, no. 1, article no. 40, 2017.

[46] G. Alon, A. F. Levitt, and P. A. McCarthy, "Functional electrical stimulation enhancement of upper extremity functional recovery during stroke rehabilitation: a pilot study," Neurorehabilitation and Neural Repair, vol. 21, no. 3, pp. 207-215, 2007.

[47] S. Mangold, C. Schuster, T. Keller, A. Zimmermann-Schlatter, and T. Ettlin, "Motor training of upper extremity with functional electrical stimulation in early stroke rehabilitation," $\mathrm{Neu}$ rorehabilitation and Neural Repair, vol. 23, no. 2, pp. 184-190, 2009.

[48] J. R. de Kroon, M. J. IJzerman, J. Chae, G. J. Lankhorst, and G. Zilvold, "Relation between stimulation characteristics and clinical outcome in studies using electrical stimulation to improve motor control of the upper extremity in stroke," Journal of Rehabilitation Medicine, vol. 37, no. 2, pp. 65-74, 2005. 
[49] D. B. Popović and M. B. Popović, "Hybrid assistive systems for rehabilitation: lessons learned from functional electrical therapy in hemiplegics," in Proceedings of the 28th Annual International Conference of the IEEE Engineering in Medicine and Biology Society, EMBS'06, vol. 1, pp. 2146-2149, September 2006.

[50] R. J. Oconnor, A. Jackson, S. G. Makower, A. Cozens, and M. Levesley, "A proof of concept study investigating the feasibility of combining iPAM robot assisted rehabilitation with functional electrical stimulation to deliver whole arm exercise in stroke survivors," Journal of Medical Engineering and Technology, vol. 39, no. 7, pp. 411-418, 2015.

[51] R. C. Lyle, "A performance test for assessment of upper limb function in physical rehabilitation treatment and research," International Journal of Rehabilitation Research, vol. 4, no. 4, pp. 483-492, 1981.

[52] M. Penta, L. Tesio, C. Arnould, A. Zancan, and J.-L. Thonnard, "The ABILHAND questionnaire as a measure of manual ability in chronic stroke patients: rasch-based validation and relationship to upper limb impairment," Stroke, vol. 32, no. 7, pp. 16271634, 2001.

[53] A. R. Fugl-Meyer, L. Jaasko, I. Leyman, S. Olsson, and S. Steglind, "The post-stroke hemiplegic patient. 1. a method for evaluation of physical performance," Scandinavian Journal of Rehabilitation Medicine, vol. 7, no. 1, pp. 13-31, 1975.

[54] K. L. Meadmore, A.-M. Hughes, C. T. Freeman et al., "Functional electrical stimulation mediated by iterative learning control and $3 \mathrm{D}$ robotics reduces motor impairment in chronic stroke," Journal of NeuroEngineering and Rehabilitation, vol. 9, no. 1, article 32, 2012.

[55] X.-L. Hu, R. K.-Y. Tong, N. S. K. Ho, J.-J. Xue, W. Rong, and L. S. W. Li, "Wrist rehabilitation assisted by an electromyographydriven neuromuscular electrical stimulation robot after stroke," Neurorehabilitation and Neural Repair, vol. 29, no. 8, pp. 767776, 2015.

[56] H. Miyasaka, A. Orand, H. Ohnishi, G. Tanino, K. Takeda, and S. Sonoda, "Ability of electrical stimulation therapy to improve the effectiveness of robotic training for paretic upper limbs in patients with stroke," Medical Engineering and Physics, vol. 38, no. 11, pp. 1172-1175, 2016.

[57] F. Resquín, A. Cuesta Gómez, J. Gonzalez-Vargas et al., "Hybrid robotic systems for upper limb rehabilitation after stroke: a review," Medical Engineering and Physics, vol. 38, no. 11, pp. 1279-1288, 2016.

[58] S. Bashir, I. Mizrahi, K. Weaver, F. Fregni, and A. Pascual-Leone, "Assessment and modulation of neural plasticity in rehabilitation with transcranial magnetic stimulation," $P M$ and $R$, vol. 2, no. 12, pp. 253-268, 2010.

[59] J. D. Schaechter, "Motor rehabilitation and brain plasticity after hemiparetic stroke," Progress in Neurobiology, vol. 73, no. 1, pp. 61-72, 2004.

[60] S. Young and K. H. Kong, "Emerging therapies in stroke rehabilitation," ANNALS Academy of Medicine Singapore, vol. 36, no. 1, pp. 58-61, 2007.

[61] M. Ameli, C. Grefkes, F. Kemper et al., "Differential effects of high-frequency repetitive transcranial magnetic stimulation over ipsilesional primary motor cortex in cortical and subcortical middle cerebral artery stroke," Annals of Neurology, vol. 66, no. 3, pp. 298-309, 2009.

[62] T. H. Emara, R. R. Moustafa, N. M. Elnahas et al., "Repetitive transcranial magnetic stimulation at $1 \mathrm{~Hz}$ and $5 \mathrm{~Hz}$ produces sustained improvement in motor function and disability after ischaemic stroke," European Journal of Neurology, vol. 17, no. 9, pp. 1203-1209, 2010.

[63] C. M. Stinear, P. A. Barber, M. Petoe, S. Anwar, and W. D. Byblow, "The PREP algorithm predicts potential for upper limb recovery after stroke," Brain, vol. 135, no. 8, pp. 2527-2535, 2012.

[64] M.-H. Milot, S. J. Spencer, V. Chan et al., "Corticospinal excitability as a predictor of functional gains at the affected upper limb following robotic training in chronic stroke survivors," Neurorehabilitation and Neural Repair, vol. 28, no. 9, pp. 819827, 2014.

[65] C. Buetefisch, R. Heger, W. Schicks, R. Seitz, and J. Netz, "Hebbian-type stimulation during robot-assisted training in patients with stroke," Neurorehabilitation and Neural Repair, vol. 25, no. 7, pp. 645-655, 2011.

[66] J. P. Lefaucheur, N. André-Obadia, A. Antal et al., "Evidencebased guidelines on the therapeutic use of repetitive transcranial magnetic stimulation (rTMS)," Clinical Neurophysiology Journal, vol. 125, no. 11, pp. 2150-2206, 2014.

[67] M. Abo, W. Kakuda, R. Momosaki et al., "Randomized, multicenter, comparative study of NEURO versus CIMT in poststroke patients with upper limb hemiparesis: The NEUROVERIFY Study," International Journal of Stroke, vol. 9, no. 5, pp. 607-612, 2014.

[68] A. Avenanti, M. Coccia, E. Ladavas, L. Provinciali, and M. G. Ceravolo, "Low-frequency rTMS promotes use-dependent motor plasticity in chronic stroke: a randomized trial," Neurology, vol. 78, no. 4, pp. 256-264, 2012.

[69] S. Etoh, T. Noma, K. Ikeda et al., "Effects of repetitive trascranial magnetic stimulation on repetitive facilitation exercises of the hemiplegic hand in chronic stroke patients," Journal of Rehabilitation Medicine, vol. 45, no. 9, pp. 843-847, 2013.

[70] S. C. B. Galvão, R. B. C. dos Santos, P. B. dos Santos, M. E. Cabral, and K. Monte-Silva, "Efficacy of coupling repetitive transcranial magnetic stimulation and physical therapy to reduce upper limb spasticity in stroke patients: a randomised controlled trial," Archives of Physical Medicine and Rehabilitation, vol. 95, no. 2, pp. 222-229, 2014.

[71] W. H. Chang, Y. H. Kim, W. K. Yoo et al., "rTMS with motor training modulates cortico-basal ganglia-thalamocortical circuits in stroke patients," Restorative Neurology and Neuroscience, vol. 30, no. 3, pp. 179-189, 2012.

[72] P. Graef, M. L. R. Dadalt, D. A. M. da Silva Rodrigués, C. Stein, and A. de Souza Pagnussat, "Transcranial magnetic stimulation combined with upper-limb training for improving function after stroke: a systematic review and meta-analysis," Journal of the Neurological Sciences, vol. 369, pp. 149-158, 2016.

[73] K. Hosomi, S. Morris, T. Sakamoto et al., "Daily repetitive transcranial magnetic stimulation for poststroke upper limb paresis in the subacute period," Journal of Stroke and Cerebrovascular Diseases, vol. 25, no. 7, pp. 1655-1664, 2016.

[74] D. J. Edwards, "On the understanding and development of modern physical neurorehabilitation methods: robotics and non-invasive brain stimulation," Journal of NeuroEngineering and Rehabilitation, vol. 6, no. 1, article 3, 2009.

[75] J. Lefaucheur, A. Antal, S. S. Ayache, D. H. Benninger, J. Brunelin, F. Cogiamanian et al., "Evidence-based guidelines on the therapeutic use of transcranial direct current stimulation (tDCS)," Clinical Neurophysiology, vol. 128, no. 1, pp. 56-92, 2017.

[76] A. Y. Kabakov, P. A. Muller, A. Pascual-Leone, F. E. Jensen, and A. Rotenberg, "Contribution of axonal orientation to pathwaydependent modulation of excitatory transmission by direct 
current stimulation in isolated rat hippocampus," Journal of Neurophysiology, vol. 107, no. 7, pp. 1881-1889, 2012.

[77] R. Lindenberg, V. Renga, L. L. Zhu, D. Nair, and G. Schlaug, "Bihemispheric brain stimulation facilitates motor recovery in chronic stroke patients," Neurology, vol. 75, no. 24, pp. 21762184, 2010.

[78] F. Hummel, P. Celnik, P. Giraux et al., "Effects of non-invasive cortical stimulation on skilled motor function in chronic stroke," Brain, vol. 128, no. 3, pp. 490-499, 2005.

[79] L. V. Bradnam, C. M. Stinear, P. A. Barber, and W. D. Byblow, "Contralesional hemisphere control of the proximal paretic upper limb following stroke," Cerebral Cortex, vol. 22, no. 11, pp. 2662-2671, 2012.

[80] D. Wu, L. Qian, R. D. Zorowitz, L. Zhang, Y. Qu, and Y. Yuan, "Effects on decreasing upper-limb poststroke muscle tone using transcranial direct current stimulation: a randomized shamcontrolled study," Archives of Physical Medicine and Rehabilitation, vol. 94, no. 1, pp. 1-8, 2013.

[81] H. Mahmoudi, A. B. Haghighi, P. Petramfar, S. Jahanshahi, Z. Salehi, and F. Fregni, "Transcranial direct current stimulation: electrode montage in stroke," Disability and Rehabilitation, vol. 33, no. 15-16, pp. 1383-1388, 2011.

[82] M. Zimerman, K. F. Heise, J. Hoppe, L. G. Cohen, C. Gerloff, and F. C. Hummel, "Modulation of training by single-session transcranial direct current stimulation to the intact motor cortex enhances motor skill acquisition of the paretic hand," Stroke, vol. 43, no. 8, pp. 2185-2191, 2012.

[83] D. J. Edwards, H. I. Krebs, A. Rykman et al., "Raised corticomotor excitability of M1 forearm area following anodal tDCS is sustained during robotic wrist therapy in chronic stroke," Restorative Neurology and Neuroscience, vol. 27, no. 3, pp. 199207, 2009.

[84] S. Hesse, A. Waldner, J. Mehrholz, C. Tomelleri, M. Pohl, and C. Werner, "Combined transcranial direct current stimulation and robot-assisted arm training in subacute stroke patients: an exploratory, randomized multicenter trial," Neurorehabilitation and Neural Repair, vol. 25, no. 9, pp. 838-846, 2011.

[85] L. T. Triccas, J. H. Burridge, A. Hughes, G. Verheyden, M. Desikan, and J. Rothwell, "A double-blinded randomised controlled trial exploring the effect of anodal transcranial direct current stimulation and uni-lateral robot therapy for the impaired upper limb in sub-acute and chronic stroke," NeuroRehabilitation, vol. 37, no. 2, pp. 181-191, 2015.

[86] D. Simonetti, L. Zollo, S. Milighetti et al., "Literature review on the effects of $\mathrm{tDCS}$ coupled with robotic therapy in post stroke upper limb rehabilitation," Frontiers in Human Neuroscience, vol. 11, article 268, 2017.

[87] V. Giacobbe, H. I. Krebs, B. T. Volpe et al., "Transcranial direct current stimulation (tDCS) and robotic practice in chronic stroke: the dimension of timing," NeuroRehabilitation, vol. 33, no. 1, pp. 49-56, 2013.

[88] S. Straudi, F. Fregni, C. Martinuzzi, C. Pavarelli, S. Salvioli, and N. Basaglia, "tDCS and robotics on upper limb stroke rehabilitation: effect modification by stroke duration and type of stroke," BioMed Research International, vol. 2016, Article ID 5068127, 8 pages, 2016.

[89] M. Ochi, S. Saeki, T. Oda, Y. Matsushima, and K. Hachisuka, "Effects of anodal and cathodal transcranial direct current stimulation combined with robotic therapy on severely affected arms in chronic stroke patients," Journal of Rehabilitation Medicine, vol. 45, no. 2, pp. 137-140, 2013.
[90] S. V. Adamovich, G. G. Fluet, E. Tunik, and A. S. Merians, "Sensorimotor training in virtual reality: a review," NeuroRehabilitation, vol. 25, no. 1, pp. 29-44, 2009.

[91] G. Saposnik and M. Levin, "Virtual reality in stroke rehabilitation: a meta-analysis and implications for clinicians," Stroke, vol. 42, no. 5, pp. 1380-1386, 2011.

[92] J. E. Deutsch, A. Brettler, C. Smith et al., "Nintendo Wii sports and Wii fit game analysis, validation, and application to stroke rehabilitation," Topics in Stroke Rehabilitation, vol. 18, no. 6, pp. 701-719, 2011.

[93] A. Viau, A. G. Feldman, B. J. McFadyen, and M. F. Levin, "Reaching in reality and virtual reality: a comparison of movement kinematics in healthy subjects and in adults with hemiparesis," Journal of Neuroengineering and Rehabilitation, vol. 1, no. 1, article 11, 2004.

[94] A. Y. Dvorkin, M. Shahar, and P. L. Weiss, "Reaching within video-capture virtual reality: using virtual reality as a motor control paradigm," Cyberpsychology and Behavior, vol. 9, no. 2, pp. 133-136, 2006.

[95] K. R. Lohse, C. G. E. Hilderman, K. L. Cheung, S. Tatla, and H. F. M. Van Der Loos, "Virtual reality therapy for adults poststroke: a systematic review and meta-analysis exploring virtual environments and commercial games in therapy," PLOS ONE, vol. 9, no. 3, Article ID e93318, 2014.

[96] K. E. Laver, S. George, S. Thomas, J. E. Deutsch, and M. Crotty, "Virtual reality for stroke rehabilitation," The Cochrane database of systematic reviews, vol. 2, p. CD008349, 2015.

[97] G. C. Palma, T. B. Freitas, G. M. Bonuzzi et al., "Effects of virtual reality for stroke individuals based on the International Classification of Functioning and Health: a systematic review," Topics in Stroke Rehabilitation, vol. 24, no. 4, pp. 269-278, 2016.

[98] A. D. Mauro, E. Carrasco, D. Oyarzun et al., "Advanced hybrid technology for neurorehabilitation: the HYPER project," Intelligent Systems Reference Library, vol. 26, pp. 89-108, 2012.

[99] G. Epelde, X. Valencia, A. Ardanza et al., "Virtual arm representation and multimodal monitoring for the upper limb robot assisted teletherapy," in Proceedings of the International Congress on Neurotechnology, Electronics and Informatics (NEUROTECHNIX'13), vol. 43, pp. 69-80, January 2013.

[100] F. Grimm and A. Gharabaghi, "Closed-loop neuroprosthesis for reach-to-grasp assistance: combining adaptive multi-channel neuromuscular stimulation with a multi-joint arm exoskeleton," Frontiers in Neuroscience, vol. 10, 2016.

[101] A. Frisoli, E. Sotgiu, C. Procopio, M. Bergamasco, B. Rossi, and C. Chisari, "Design and implementation of a training strategy in chronic stroke with an arm robotic exoskeleton," in Proceedings of the Rehab Week Zurich 2011 - 2011 IEEE International Conference on Rehabilitation Robotics, ICORR 2011, vol. 2011, July 2011.

[102] A. Frisoli, L. Borelli, A. Montagner et al., "Arm rehabilitation with a robotic exoskeleleton in Virtual Reality," in Proceedings of the 2007 IEEE 10th International Conference on Rehabilitation Robotics, ICORR '07, pp. 631-642, nld, June 2007.

[103] C. D. Takahashi, L. Der-Yeghiaian, V. Le, R. R. Motiwala, and S. C. Cramer, "Robot-based hand motor therapy after stroke," Brain, vol. 131, no. 2, pp. 425-437, 2008.

[104] A. S. Merians, E. Tunik, and S. V. Adamovich, "Virtual reality to maximize function for hand and arm rehabilitation: exploration of neural mechanisms," Studies in Health Technology and Informatics, vol. 145, pp. 109-125, 2009.

[105] K. O. Thielbar, T. J. Lord, H. C. Fischer et al., "Training finger individuation with a mechatronic-virtual reality system leads to 
improved fine motor control post-stroke," Journal of NeuroEngineering and Rehabilitation, vol. 11, no. 1, article no. 690, 2014.

[106] A. Maris, K. Coninx, H. Seelen et al., "The impact of robotmediated adaptive I-TRAVLE training on impaired upper limb function in chronic stroke and multiple sclerosis," Disability and Rehabilitation: Assistive Technology, pp. 1-9, 2017.

[107] D. M. Simpson, A. Blitzer, A. Brashear et al., "Assessment: botulinum neurotoxin for the treatment of movement disorders (an evidence-based review): report of the Therapeutics and Technology Assessment Subcommittee of the American Academy of Neurology," Neurology, vol. 70, no. 19, pp. 16991706, 2008.

[108] L. Shaw, Rodgers, C. Price et al., "BoTULS: a multicentre randomised controlled trial to evaluate the clinical effectiveness and cost-effectiveness of treating upper limb spasticity due to stroke with botulinum toxin type A," Health Technology Assessment, vol. 14, no. 26, pp. 1-113, 2010.

[109] J.-M. Gracies, A. Brashear, R. Jech et al., "Safety and efficacy of abobotulinumtoxinA for hemiparesis in adults with upper limb spasticity after stroke or traumatic brain injury: a double-blind randomised controlled trial," The Lancet Neurology, vol. 14, no. 10, article no. 148, pp. 992-1001, 2015.

[110] Y. Dong, T. Wu, X. Hu, and T. Wang, "Efficacy and safety of Botulinum Toxin type A for upper limb spasticity after stroke or traumatic brain injury: a systematic review with meta-analysis and trial sequential analysis," European Journal of Physical and Rehabilitation Medicine, 2016.

[111] M. Vinti, F. Costantino, N. Bayle, D. M. Simpson, D. J. Weisz, and J.-M. Gracies, "Spastic cocontraction in hemiparesis: effects of botulinum toxin," Muscle and Nerve, vol. 46, no. 6, pp. 926931, 2012.

[112] F. I. Mahoney and D. W. Barthel, "Functional evaluation: the Barthel Index," Maryland State Medical Journal, vol. 14, pp. 6165, 1965.

[113] M. Beninato, K. M. Gill-Body, S. Salles, P. C. Stark, R. M. BlackSchaffer, and J. Stein, "Determination of the minimal clinically important difference in the FIM instrument in patients with stroke," Archives of Physical Medicine and Rehabilitation, vol. 87, no. 1, pp. 32-39, 2006.

[114] C. Anderson, S. Laubscher, and R. Burns, "Validation of the short form 36 (SF-36) health survey questionnaire among stroke patients," Stroke, vol. 27, no. 10, pp. 1812-1816, 1996.

[115] R. L. Rosales, F. Efendy, E. S. Teleg et al., "Botulinum toxin as early intervention for spasticity after stroke or non-progressive brain lesion: a meta-analysis," Journal of the Neurological Sciences, vol. 371, pp. 6-14, 2016.

[116] S. Hesse and C. Werner, "Poststroke motor dysfunction and spasticity: novel pharmacological and physical treatment strategies," CNS Drugs, vol. 17, no. 15, pp. 1093-1107, 2003.

[117] F. Posteraro, S. Mazzoleni, S. Aliboni et al., "Upper limb spasticity reduction following active training: a robot-mediated study in patients with chronic hemiparesis," Journal of Rehabilitation Medicine, vol. 42, no. 3, pp. 279-281, 2010.

[118] P. Sale, M. Franceschini, S. Mazzoleni, E. Palma, M. Agosti, and F. Posteraro, "Effects of upper limb robot-assisted therapy on motor recovery in subacute stroke patients," Journal of NeuroEngineering and Rehabilitation, vol. 11, no. 1, article 104, 2014.

[119] F. Bovolenta, P. Sale, V. Dall'Armi, P. Clerici, and M. Franceschini, "Robot-aided therapy for upper limbs in patients with stroke-related lesions. Brief report of a clinical experience,"
Journal of NeuroEngineering and Rehabilitation, vol. 8, no. 1, article no. 18, 2011.

[120] A. C. Lo, Guarino P. D., L. G. Richards et al., "Robot-assisted therapy for long-term upper-limb impairment after stroke," The New England Journal of Medicine, vol. 362, no. 19, pp. 1772-1783, 2010.

[121] S. E. Fasoli, M. Fragala-Pinkham, R. Hughes, N. Hogan, H. I. Krebs, and J. Stein, "Upper limb robotic therapy for children with hemiplegia," American Journal of Physical Medicine and Rehabilitation, vol. 87, no. 11, pp. 929-936, 2008.

[122] F. Frascarelli, L. Masia, G. Di Rosa, M. Petrarca, P. Cappa, and E. Castelli, "Robot-mediated and clinical scales evaluation after upper limb botulinum toxin type A injection in children with hemiplegia," Journal of Rehabilitation Medicine, vol. 41, no. 12, pp. 988-994, 2009.

[123] T. Takebayashi, S. Amano, K. Hanada et al., "Therapeutic synergism in the treatment of post-stroke arm paresis utilizing botulinum toxin, robotic therapy, and constraint-induced movement therapy," PM and R, vol. 6, no. 11, pp. 1054-1058, 2014.

[124] G. V. Pennati, C. Da Re, I. Messineo, and D. Bonaiuti, "How could robotic training and botolinum toxin be combined in chronic post stroke upper limb spasticity? A pilot study," European Journal of Physical and Rehabilitation Medicine, vol. 51, no. 4, pp. 381-387, 2015.

[125] K. Saita, T. Morishita, K. Hyakutake et al., "Combined therapy using botulinum toxin A and single-joint hybrid assistive limb for upper-limb disability due to spastic hemiplegia," Journal of the Neurological Sciences, vol. 373, pp. 182-187, 2017.

[126] D. L. Turner, A. Ramos-Murguialday, N. Birbaumer, U. Hoffmann, and A. Luft, "Neurophysiology of robot-mediated training and therapy: a perspective for future use in clinical populations," Frontiers in Neurology, vol. 4, article 184, 2013.

[127] C. M. Stinear, P. A. Barber, J. P. Coxon, M. K. Fleming, and W. D. Byblow, "Priming the motor system enhances the effects of upper limb therapy in chronic stroke," Brain, vol. 131, no. 5, pp. 1381-1390, 2008.

[128] V. Di Lazzaro, F. Capone, G. Di Pino et al., "Combining robotic training and non-invasive brain stimulation in severe upper limb-impaired chronic stroke patients," Frontiers in Neuroscience, vol. 10, article no. 88, 2016.

[129] K. M. Malloy and L. S. Milling, "The effectiveness of virtual reality distraction for pain reduction: a systematic review," Clinical Psychology Review, vol. 30, no. 8, pp. 1011-1018, 2010.

[130] G. Rosati, F. Oscari, D. J. Reinkensmeyer et al., "Improving robotics for neurorehabilitation: Enhancing engagement, performance, and learning with auditory feedback," in Proceedings of the Rehab Week Zurich 2011 - 2011 IEEE International Conference on Rehabilitation Robotics, ICORR '11, vol. 2011, July 2011.

[131] R. Secoli, M.-H. Milot, G. Rosati, and D. J. Reinkensmeyer, "Effect of visual distraction and auditory feedback on patient effort during robot-assisted movement training after stroke," Journal of NeuroEngineering and Rehabilitation, vol. 8, no. 1, article no. 21, 2011.

[132] M. C. Cirstea and M. F. Levin, "Improvement of arm movement patterns and endpoint control depends on type of feedback during practice in stroke survivors," Neurorehabilitation and Neural Repair, vol. 21, no. 5, pp. 398-411, 2007.

[133] L. Dipietro, H. I. Krebs, S. E. Fasoli et al., "Changing motor synergies in chronic stroke," Journal of Neurophysiology, vol. 98, no. 2, pp. 757-768, 2007. 
[134] N. Hogan, H. I. Krebs, B. Rohrer et al., "Motions or muscles? Some behavioral factors underlying robotic assistance of motor recovery," The Journal of Rehabilitation Research and Development, vol. 43, no. 5, pp. 605-618, 2006.

[135] H. I. Krebs, S. Mernoff, S. E. Fasoli, R. Hughes, J. Stein, and N. Hogan, "A comparison of functional and impairment-based robotic training in severe to moderate chronic stroke: a pilot study," NeuroRehabilitation, vol. 23, no. 1, pp. 81-87, 2008.

[136] M. Jeannerod, "The origin of voluntary action. History of a physiological concept," Comptes Rendus - Biologies, vol. 329, no. 5-6, pp. 354-362, 2006.

[137] A. A. Blank, J. A. French, A. U. Pehlivan, and M. K. O’Malley, "Current trends in robot-assisted upper-limb stroke rehabilitation: promoting patient engagement in therapy," Current Physical Medicine and Rehabilitation Reports, vol. 2, no. 3, pp. 184-195, 2014.

[138] W. Schultz, "Behavioral theories and the neurophysiology of reward," Annual Review of Psychology, vol. 57, pp. 87-115, 2006.

[139] P. Ramkumar, B. Dekleva, S. Cooler, L. Miller, and K. Kording, "Premotor and motor cortices encode reward," PLOS ONE, vol. 11, no. 8, Article ID e0160851, 2016.

[140] R. N. Goodman, J. C. Rietschel, A. Roy et al., "Increased reward in ankle robotics training enhances motor control and cortical efficiency in stroke," Journal of Rehabilitation Research and Development, vol. 51, no. 2, pp. 213-227, 2014.

[141] E. A. A. Rameckers, L. A. W. M. Speth, J. Duysens, J. S. H. Vles, and B. C. M. Smits-Engelsman, "Kinematic aiming task: measuring functional changes in hand and arm movements after botulinum toxin-A injections in children with spastic hemiplegia," American Journal of Physical Medicine and Rehabilitation, vol. 86, no. 7, pp. 538-547, 2007.

[142] A. H. Mackey, F. Miller, S. E. Walt, M.-C. Waugh, and N. S. Stott, "Use of three-dimensional kinematic analysis following upper limb botulinum toxin a for children with hemiplegia," European Journal of Neurology, vol. 15, no. 11, pp. 1191-1198, 2008.

[143] E. A. Fridman, M. Crespo, S. G. Argüello et al., "Kinematic improvement following Botulinum Toxin-A injection in upperlimb spasticity due to stroke," Journal of Neurology, Neurosurgery and Psychiatry, vol. 81, no. 4, pp. 423-427, 2010.

[144] D. Bensmail, J. V. G. Robertson, C. Fermanian, and A. RobyBrami, "Botulinum toxin to treat upper-limb spasticity in hemiparetic patients: analysis of function and kinematics of reaching movements," Neurorehabilitation and Neural Repair, vol. 24, no. 3, pp. 273-281, 2010. 


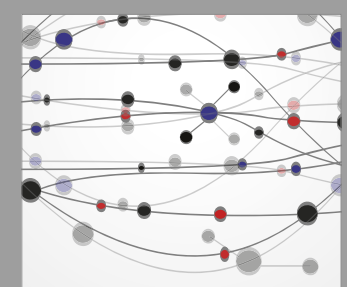

The Scientific World Journal
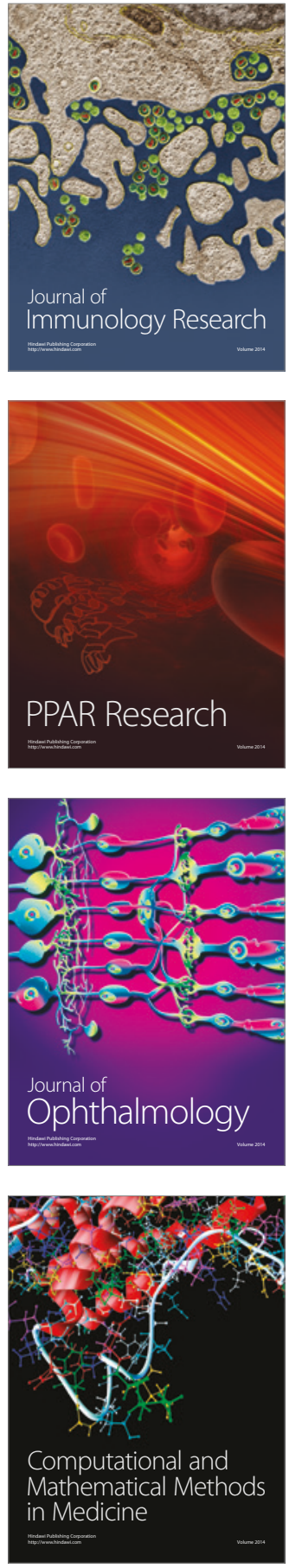

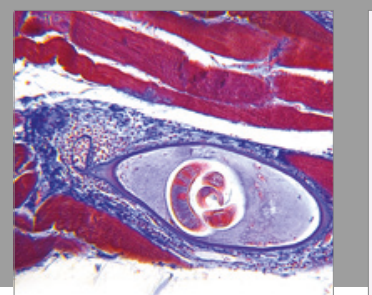

Gastroenterology Research and Practice
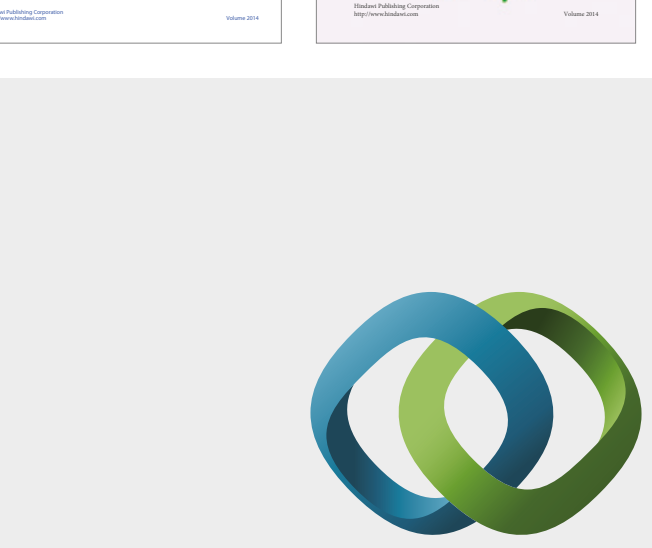

\section{Hindawi}

Submit your manuscripts at

https://www.hindawi.com
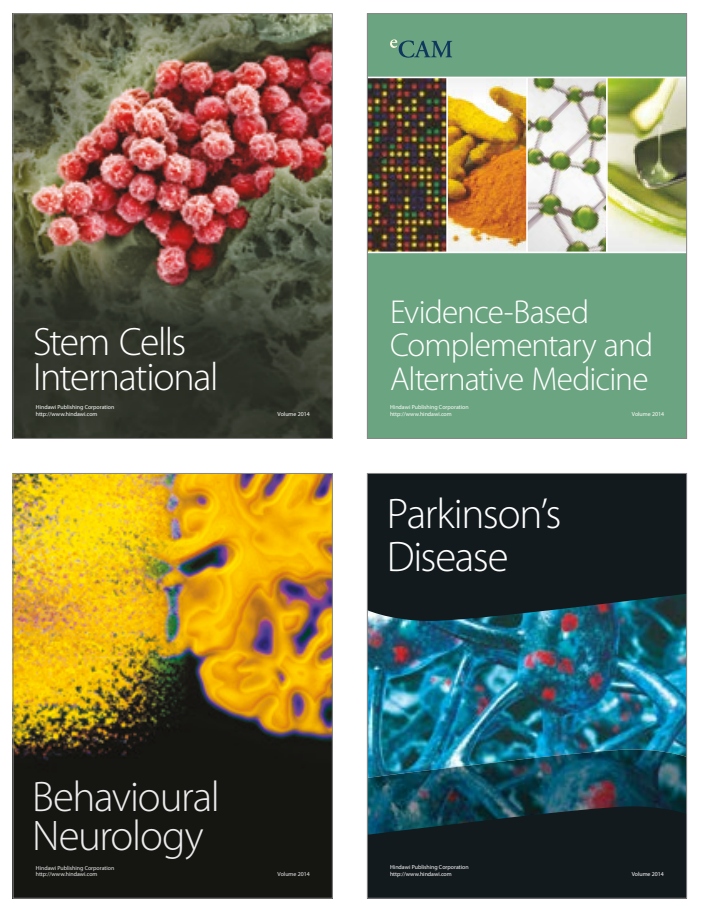
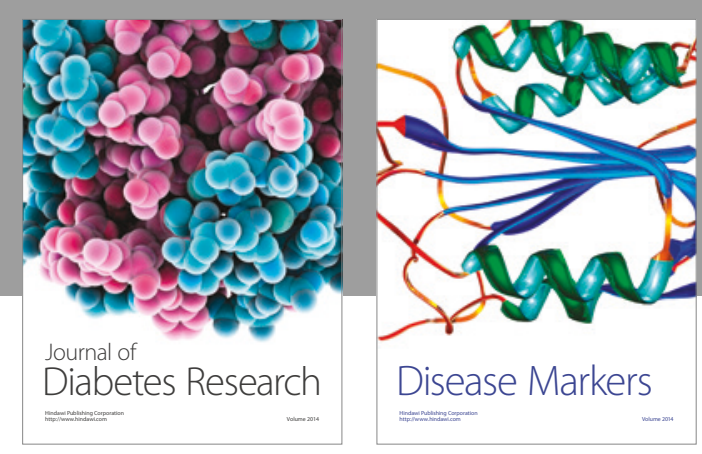

Disease Markers
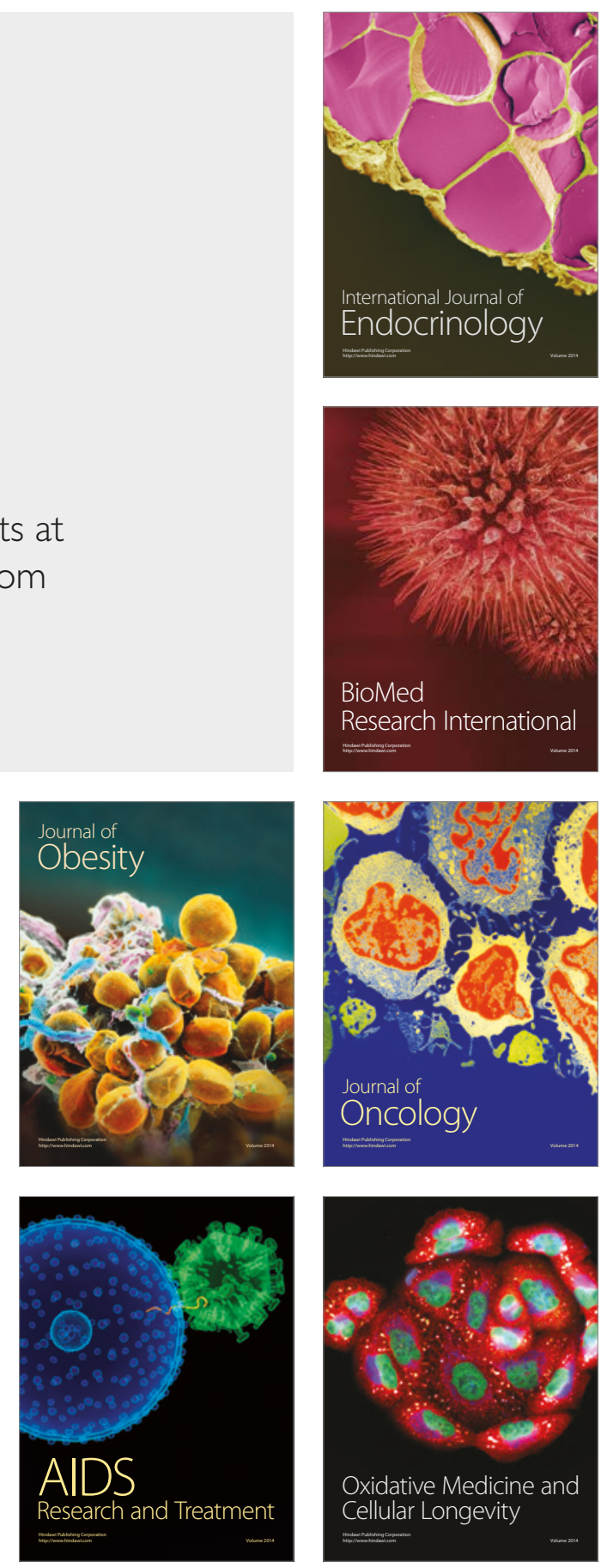